\title{
Recomendaciones de diseño y construcción de pavimentos en placa-huella de concreto reforzado
}

\author{
Armando Orobio* \\ Juan Carlos Orobio** \\ Juan Manuel Mosquera ${ }^{* * *}$
}

Recibido: 29/01/2016 • Aceptado: 21/07/2017

DOI: 10.22395/rium.v17n32a4

\section{Resumen}

La construcción de pavimentos en placa-huella de concreto reforzado se ha incrementado en Colombia en los últimos años. La gran mayoría de los proyectos se basa en la especificación 500-1P que presenta un diseño general, del cual se desconocen los criterios tenidos en cuenta en su elaboración, lo que genera muchas dudas para su uso en diferentes proyectos. En el presente artículo se hace un análisis de los pavimentos en placa-huella, y se dan recomendaciones de diseño y construcción de este tipo de pavimentos, considerando las condiciones de uso actual, las cargas esperadas, las características de suelo de soporte y las calidades de los materiales.

Palabras clave: pavimentos de concreto; placa-huella; construcción; diseño.

Profesor titular, Escuela de Ingeniería Civil y Geomática, Universidad del Valle, Colombia. armando.orobio@ correounivalle.edu.co

** Director de Proyectos, El Alcázar SAS, Colombia. jorobio1@gmail.com

*** Profesor, Universidad del Cauca, Colombia. mori@unicauca.edu.co 


\title{
Recommendations for the Design and Construction of Reinforced Concrete Strip Road Pavements
}

\begin{abstract}
The construction of reinforced concrete strip road pavements has increased in Colombia in recent years. The vast majority of the projects are based on the 500-1P specification, which presents a general design, of which the criteria taken into account in its elaboration are unknown, which generates many doubts for its use in different projects. In the present article, an analysis of strip road pavement is made and recommendations are given for the design and construction of this type of pavement, taking into account current conditions of use, expected loads, characteristics of the supporting soil, and the quality of materials.
\end{abstract}

Keywords: concrete pavements; strip road pavement; construction; design.

\section{Recomendações de desenho e construção de pavimentos em placa de concreto armado}

\begin{abstract}
Resumo
A construção de pavimentos em placa de concreto armado tem aumentado na Colômbia nos últimos anos. A grande maioria dos projetos baseia-se na especificação 500-1P, que apresenta um desenho geral, do qual se desconhecem os critérios considerados em sua elaboração, o que gera muitas dúvidas para seu uso em diferentes projetos. Neste artigo, faz-se uma análise dos trilhos em placa de concreto e dão-se recomendações de desenho e construção desse tipo de pavimentos, levando em conta as condições de uso atual, as cargas esperadas, as características de solo de suporte e a qualidade dos materiais.
\end{abstract}

Palavras-chave: pavimentos de concreto; placa-trilho; construção; desenho. 


\section{INTRODUCCIÓN}

La placa-huella es un sistema de pavimentación para vías de bajo volumen de tránsito, en el cual se pavimenta en concreto, con refuerzo continuo, únicamente la franja por la que circulan las ruedas de los vehículos (figura 1) [1]. Las placas son conectadas entre sí con una riostra en concreto reforzado que une las dos placas y las bermas-cuneta, con el fin que estas funcionen monolíticamente. Los espacios entre las placas-huella, las riostras y las bermas-cuneta se rellenan con una capa de concreto ciclópeo del mismo espesor de la placa-huella. Los pavimentos en placa-huella se consideran una alternativa económica para redes terciarias con bajo volumen de tránsito.

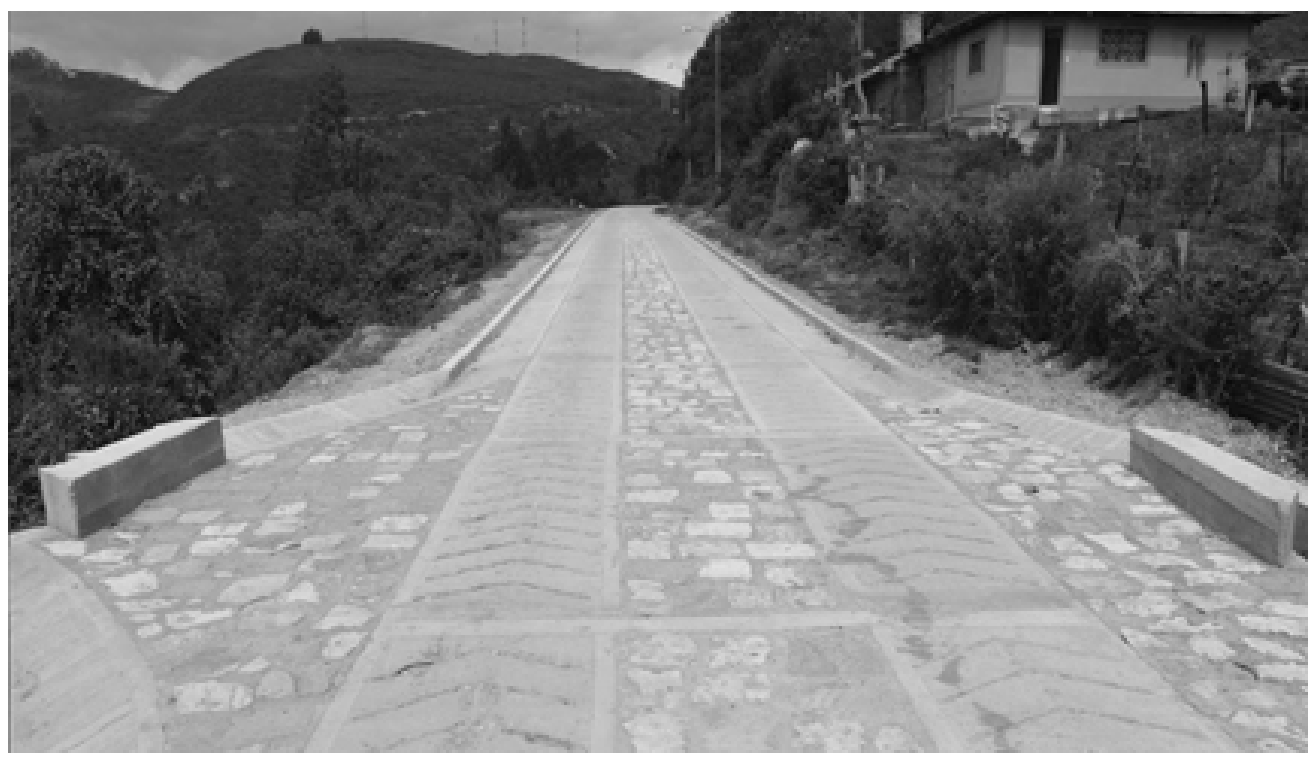

Figura 1. Placa-huella en concreto reforzado Fuente: [1]

Debido a la creciente utilización de pavimentos en placa-huella en la red vial nacional en los últimos años, lo cual ha permitido mejorar la superficie de rodadura y reducir costos de mantenimiento de vías terciarias en muchas regiones del país, el Instituto Nacional de Vías contrató mediante concurso de méritos al Consorcio Manuales y Guías Ceal, conformado por las firmas El Alcázar SAS, Compañía de Consultoría y Construcciones Ltda., y Vías Alfa E. U., para la elaboración de una Guía de Diseño de Pavimentos con Placa Huella. Dentro de los requisitos contractuales se requiere un período de diseño de 20 años para los pavimentos en placa-huella y la estandarización de esta tecnología para un mejor uso en Colombia. En este artículo se presentan algunos de los análisis realizados para el diseño estructural de los pavimentos en placa-huella y se suministran recomendaciones para la construcción de este tipo de pavimentos. 


\section{SITUACIÓN ACTUAL DE LOS PAVIMENTOS EN PLACA-HUELLA EN COLOMBIA}

A pesar del auge que actualmente tienen los pavimentos en placa-huella, que son construidos por la Subdirección de Red Terciaria del Instituto Nacional de Vías (Invías), gobernaciones de departamentos y alcaldías municipales, actualmente no existe una guía de diseño y construcción para este tipo de pavimentos. La información más completa que se encuentra disponible es la especificación particular 500-1P [2] de la cual no existe mayor información, se desconoce su origen y al parecer no es una especificación oficial del Invías, sin embargo, se ha venido utilizando en ausencia de una mejor especificación.

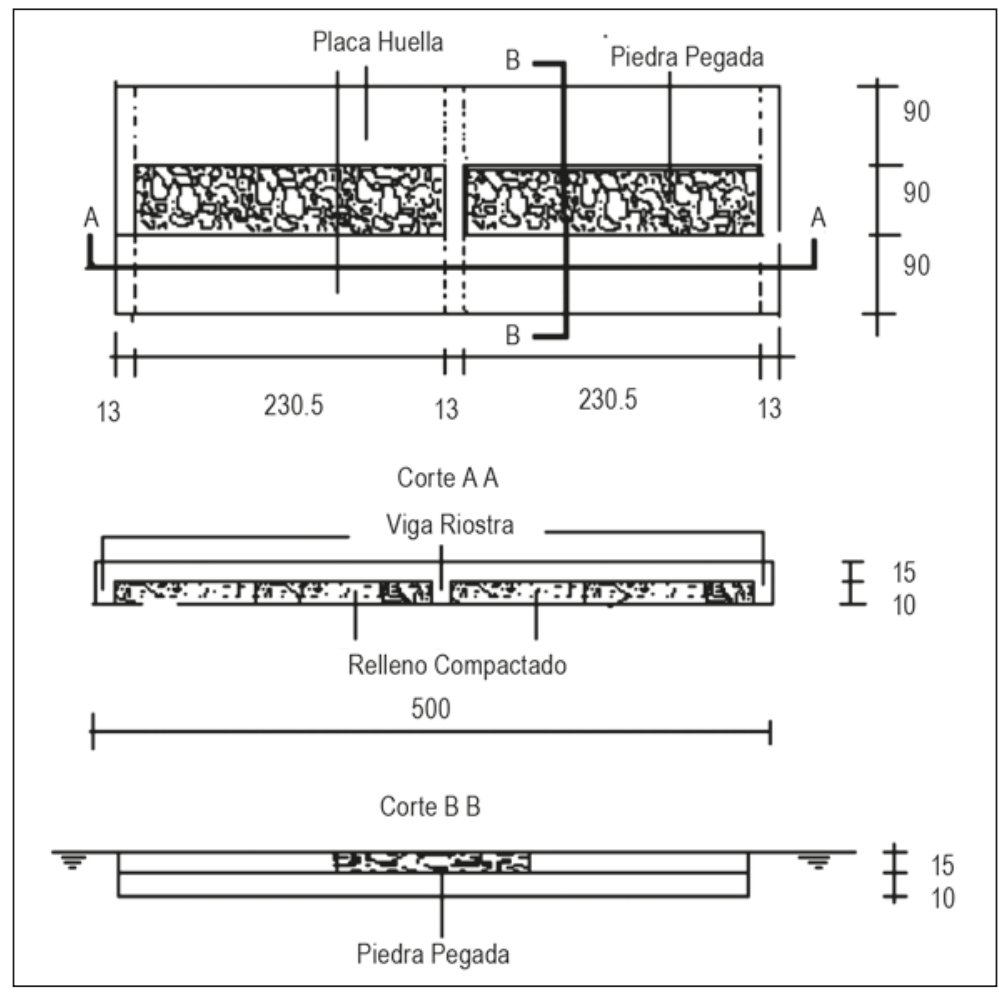

Figura 2. Perfil-del-suelo-estudiado

Fuente: elaboración propia

En la figura 2 se muestra la geometría de los pavimentos en placa-huella según la especificación 500-1P; el pavimento consiste en dos placas-huella paralelas en concreto reforzado. La figura muestra placas-huella en concreto reforzado de $0,90 \mathrm{~m}$ de ancho y 5,0 m de longitud, con riostras reforzadas en los extremos y en el centro de la longitud, con sección de $0,13 \mathrm{~m} \times 0,25 \mathrm{~m}$. La placa-huella tiene un espesor de 0,15 $\mathrm{m}$ y está apoyada sobre un material de afirmado de $0,10 \mathrm{~m}$ de espesor que suministra 
un adecuado soporte a la placa-huella. En la especificación 500-1P el refuerzo está definido como una armadura de acero en dos direcciones con barras \#2 (1/4") separadas cada $0,30 \mathrm{~m}$. Las riostras requieren dos aceros \#3 (3/8") en la parte superior, la misma cuantía de refuerzo en la parte inferior y estribos \#2 (1/4") cada 0,20 m.

Sin embargo, no se conocen memorias de cálculo, no se sabe con qué criterios de carga se llegó al diseño propuesto, ni cuáles fueron las condiciones geotécnicas consideradas para el soporte. Un gran interrogante es si con las características actuales, los pavimentos en placa-huella están en capacidad de tener un buen desempeño durante un período de diseño de 20 años, con las cargas vehiculares esperadas.

En la práctica, cuando se revisan proyectos construidos, con frecuencia se encuentran modificaciones a lo dispuesto en la especificación 500-1P; se incrementa el espesor de la placas-huella, pero con la misma cuantía de acero de refuerzo, se aumentan las dimensiones de las riostras y se incrementan los espesores de material de soporte, cambios del lado de la seguridad, seguramente debidos a la falta de información de los criterios que dieron origen al diseño presentado en la especificación 500-1P, pero que se hacen con poco rigor técnico, debido a que no se analiza el comportamiento de las placas-huella con consideraciones de geometría, cargas de tránsito, capacidad de soporte del suelo y clima.

En la presente investigación se analizó el comportamiento de los pavimentos en placa-huella con losas continuamente reforzadas. El análisis basado en la teoría de Winkler para calcular deflexiones, cortantes y momentos en vigas apoyadas sobre fundaciones elásticas, se realizó mediante un modelo de elementos finitos para evaluar la respuesta de las placas-huella a las cargas críticas del tránsito, con diferentes calidades de apoyo, y proponer un diseño que se ajuste a las condiciones de uso nacionales. Al final se realizan comparaciones con la especificación 500-1P y se hacen recomendaciones constructivas.

\section{DESCRIPCIÓN DEL MODELO}

El modelo se basó en la teoría de Winkler para el análisis de vigas sobre fundaciones elásticas, que consiste en representar la viga soportada sobre numerosos resortes de constante $\mathrm{k}$ y sometida a unas condiciones de carga (figura 3a); en la figura, Q representa la carga aplicada sobre la viga, en este caso. En el diagrama de cuerpo libre (figura $3 b)$, se observan el esfuerzo cortante $(V)$, el momento $(M)$ y la reacción de los resortes, con una longitud $d x$, que se representa por $k w d x$, donde $w$ es el desplazamiento de la viga en dirección $Z$ [3]. 


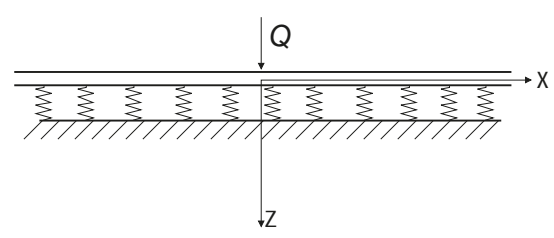

a)

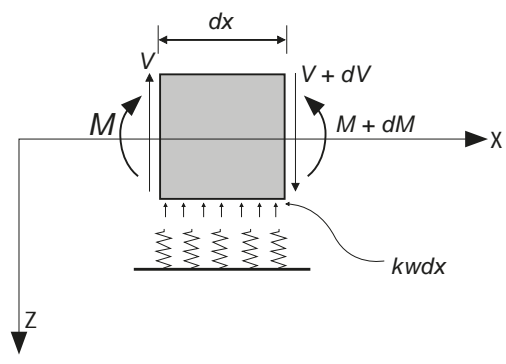

b)

Figura 3. Viga soportada en una fundación elástica

Fuente: [3]

El equilibrio de fuerzas está dado por la ecuación 1, y del equilibrio de momentos se obtiene la ecuación 2. Reemplazando la ecuación 2 en la ecuación 1 y haciendo $E I \frac{d^{4} w}{d x^{4}}=-M$ (Euler-Bernoulli) se obtiene la ecuación 3. Donde EI es la rigidez de la viga.

$$
\begin{gathered}
V-(V+d V)+k w d x=0 \\
V=\frac{d M}{d x} \\
E I \frac{d^{4} w}{d x^{4}}-k w=0
\end{gathered}
$$

La ecuación 3 es utilizada en ingeniería para representar vigas soportadas sobre fundaciones elásticas; la solución general se presenta en la ecuación 4 [3], donde $\lambda=\left(\frac{k}{4 E I}\right)^{\frac{1}{4}}, C_{1}, C_{2}, C_{3} \mathrm{y} C_{4}$ son constantes, las cuales pueden ser determinadas a partir de las condiciones de frontera del problema específico.

$$
w=e^{\lambda x}\left(C_{1} \cos \lambda x+C_{2} \sin \lambda x\right)+e^{-\lambda x}\left(C_{3} \cos \lambda x+C_{4} \sin \lambda x\right)
$$

La condición $\lim _{x \rightarrow \infty} w=0$ conlleva a que $C_{1}=C_{2}=0$, por simetría $\left.\frac{d w}{d x}\right|_{x \rightarrow 0}=0$, lo que conlleva a que $C_{3}=C_{4}=C$, realizando sumatoria de fuerzas verticales $2 \int_{0}^{\infty} k w d x=Q$, lo que implica que $C=\frac{Q \lambda}{2 k}$; en consecuencia, la deflexión en una viga soportada en 
una fundación elástica se obtiene de la ecuación 5 para $x \geq 0$ y de la ecuación 6 para $x<0$. Con diferenciación sucesiva se pueden obtener la rotación, el momento flector y el esfuerzo cortante [3]. Detalles de la solución de la ecuación diferencial pueden consultarse en la referencia [4].

$$
\begin{gathered}
w=\frac{Q \lambda}{2 k} e^{-\lambda x}(\cos \lambda x+\sin \lambda x) \\
w=\frac{Q \lambda}{2 k} e^{\lambda x}(\cos \lambda x-\sin \lambda x)
\end{gathered}
$$

Para el caso del presente estudio, el modelo de Winkler se utilizó para determinar los diagramas de deformaciones (w), momento (M) y cortante (V) que se presentan en los elementos del pavimento en placa-huella. Se estructuró un modelo de elementos finitos de una placa huella con riostras separadas cada tres metros (figura 4). El modelo consistió en 7 módulos de 3,0 m cada uno, con el fin de representar la continuidad de la estructura; las cargas se ubicaron en el módulo central, el cual, a su vez, se dividió con diferentes nodos internos para poder determinar los esfuerzos máximos generados por el paso del vehículo de diseño. El modelo consideró que la placa huella se encuentra apoyada sobre una capa granular continua de soporte.

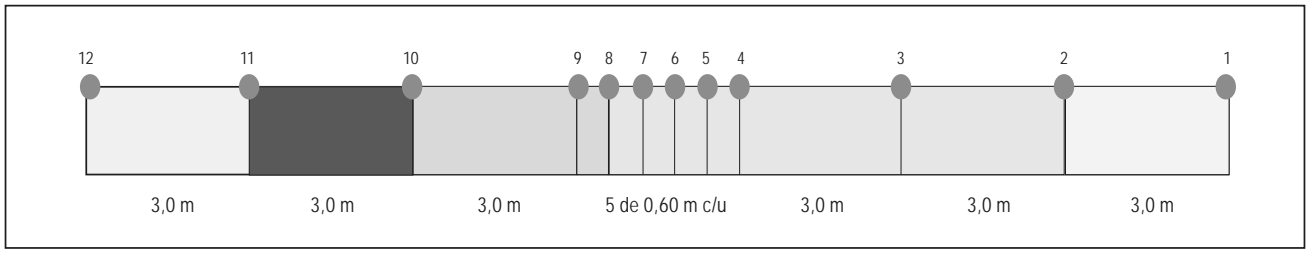

Figura 4. Modelo para evaluación de la placa-huella.

Fuente: elaboración propia

Los nodos del modelo se dividieron en tres tipos:

- Nodos externos $(1,2,3,10,11$ y 12) los cuales tienen como función representar la continuidad de la estructura.

- Nodos internos-externos $(9,4)$ que son los extremos del tramo cargado

- Nodos internos $(8,7,6$ y 5) los cuales corresponden a puntos de ubicación de las cargas.

\section{DETERMINACIÓN DE LA CARGA APLICADA A LA PLACA-HUELLA}

Con el fin de brindar un grado adecuado de confiabilidad al modelo planteado, las cargas fueron mayoradas para garantizar que los supuestos del modelo se cumplirán 
durante la fase de operación de la placa huella. Teniendo en cuenta que las inversiones en pavimentación de vías son cuantiosas y que se trata de la elaboración de un diseño de aplicación general, con un período de diseño de 20 años, se considera que el grado de confiabilidad debe ser alto.

\subsection{Factores de mayoración de cargas}

Para obtener un grado de confiabilidad alto, la determinación de las cargas a considerar en el diseño de pavimentos con placa-huella debe minimizar las posibilidades de falla por incertidumbre en la estimación de cargas. Ante la poca información existente sobre factores de mayoración de cargas para el diseño de pavimentos reforzados, se siguió la recomendación de Arango-Londoño [5], que propone la utilización de los siguientes factores de mayoración:

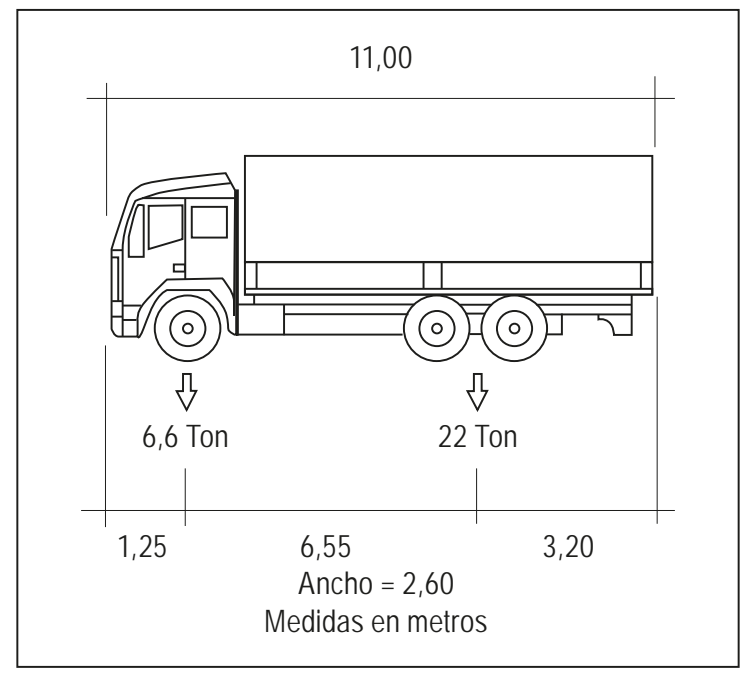

Figura 5. Pesos y dimensiones del vehículo de diseño-C3 Fuente: [6]

- Factor de mayoración para carga muerta $\quad 1,50$

- Factor de mayoración para carga viva $\quad 1,75$

\subsection{Vehículo de diseño}

Los pavimentos en placa huella son utilizados para vías de bajos volúmenes de tránsito en vías de la red terciaria y secundaria. Los vehículos de mayor peso que se espera circulen por este tipo de pavimentos corresponden al camión C3, el cual tiene un eje simple de rueda simple adelante (SRS), y un eje tándem atrás (figura 5). Los 
pesos máximos para estos ejes están regulados por la resolución 004100 del 2004 del Ministerio de Transporte [6], por lo cual se usa el peso máximo permitido para efecto de los análisis en el presente estudio. La distribución de cargas entre los lados del eje se consideró del 50\%; en pavimentos de placa-huella cada una de las huellas de concreto

El camión C3 en su parte trasera tiene un eje tándem compuesto por dos ejes simples de rueda doble, separados 1,2 metros entre centros. Con el fin de representar de manera más real la aplicación de esta carga, se considera una distribución uniforme entre las ruedas del semieje, como se muestra en la figura 6 .

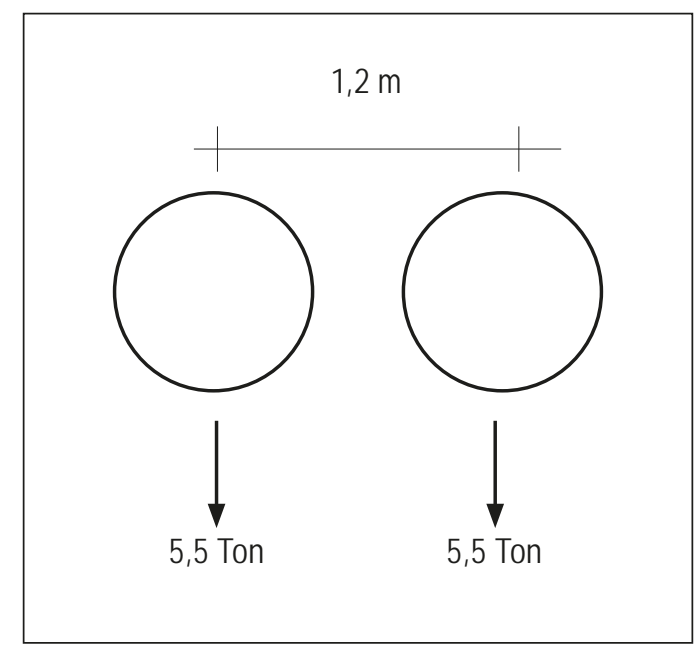

Figura 6. Distribución de carga en el semieje tándem del camión C3.

Fuente: elaboración propia

Con el factor de mayoración de carga viva definido anteriormente, la carga viva a utilizar para cada rueda corresponde a 9,63 t $(96,3 \mathrm{kN})$; para efecto de simplicidad se adoptó una carga de diseño $9,5 \mathrm{t}(95 \mathrm{kN})$ para cada rueda del semieje tándem.

\subsection{Carga por peso propio}

Las cargas derivadas del peso propio corresponden únicamente al peso del concreto que conforma la placa-huella y la riostra. Estas cargas se determinan a partir de la siguiente expresión:

Carga $=$ Factos de mayoración $*$ Volumen $*$ Peso específico del concreto. 
- Determinación de la carga por peso propio de la riostra

Se considera un elemento de 0,20 m de ancho, 0,20 m de alto y una longitud de $0,90 \mathrm{~m}$, correspondiente al ancho de la placa-huella. El peso propio mayorado de la riostra corresponde a $130 \mathrm{~kg}(1,3 \mathrm{kN})$.

\section{- Determinación de la carga por peso propio de la placa-huella}

La carga por peso propio de la placa-huella varía dependiendo de la ubicación de la losa y del tipo de nodo que le corresponda, dado que la longitud de placa que contribuye al peso que soportará cada nodo varía de la siguiente manera:

- Nodos externos

La longitud de placa corresponde a 3,0 $\mathrm{m}$ y su peso propio mayorado es de $1.460 \mathrm{~kg}(14,6 \mathrm{kN})$.

- Nodos internos-externos

La longitud de placa corresponde a 1,5 m y su peso propio mayorado es de 730 $\operatorname{kg}(7,3 \mathrm{kN})$.

- Nodos internos

La longitud de placa corresponde a 0,60 metros y su peso propio mayorado es de $290 \mathrm{~kg}(2,90 \mathrm{kN})$.

\section{CARACTERIZACIÓN DEL SOPORTE DE LA PLACA HUELLA}

De acuerdo con la experiencia existente en la construcción de los pavimentos en placa-huella reforzada, se considera que estos pavimentos tienen una superficie de soporte conformada por una capa de material granular de sub-base apoyada sobre la sub-rasante; esta capa brinda un apoyo uniforme a la placa permitiendo la disminución de esfuerzos y deformaciones.

Para efectos de determinar la calidad del apoyo se empleó la siguiente correlación del CBR con el módulo de reacción de la subrasante (ecuación 7) [7]. Con base en la ecuación 7 [8] y las correlaciones de la PCA entre el módulo resiliente y el módulo de reacción de la subrasante [9] se construyó la tabla 1.

$$
M_{R}=1.500(C B R)
$$

Donde,

$M_{R}$ : Módulo resiliente de la subrasante en psi

CBR: Capacidad de soporte de la subrasante 
Tabla 1. Correlación entre el módulo de reacción de la sub-rasante y el CBR del suelo.

\begin{tabular}{|c|c|c|c|}
\hline CBR & $M r[P S I]$ & $K[P S I]$ & $K\left[\mathrm{kN} / \mathrm{m}^{3}\right]$ \\
\hline 3 & 4.500 & 100 & 27.130 \\
\hline 4 & 6.000 & 132 & 35.812 \\
\hline 5 & 7.500 & 143 & 38.796 \\
\hline 6 & 9.000 & 152 & 41.238 \\
\hline 7 & 10.500 & 161 & 43.679 \\
\hline 8 & 12.000 & 178 & 48.291 \\
\hline 9 & 13.500 & 193 & 52.361 \\
\hline 10 & 15.000 & 200 & 54.260 \\
\hline
\end{tabular}

Fuente: elaboración propia

El aporte de la capa granular se estableció a partir de la correlación recomendada por la PCA, mostrada en la tabla 2, que considera el efecto sobre el módulo de reacción de una sub-base sin tratar colocada sobre la sub-rasante [9].

Tabla 2. Módulo de reacción del conjunto sub-rasante - sub-base [kN/m3] (Huaung, 2004 [8], Sistema de unidades modificado)

\begin{tabular}{|c|c|c|c|c|}
\hline \multirow{2}{*}{$\begin{array}{c}\text { K subrasante } \\
{\left[\mathrm{kN} / \mathrm{m}^{3}\right]}\end{array}$} & \multicolumn{4}{|c|}{ Espesor de subbase en $(\mathrm{m})$} \\
\cline { 2 - 5 } & 0,10 & 0,15 & 0,22 & 0,30 \\
\hline 13.565 & 17.635 & 20.348 & 23.061 & 29.843 \\
\hline 27.130 & 35.269 & 37.982 & 43.408 & 51.547 \\
\hline 54.260 & 59.686 & 52.399 & 73.251 & 86.816 \\
\hline 81.390 & 86.816 & 89.529 & 100.381 & 116.569 \\
\hline \multicolumn{5}{|c|}{ Fuente: elaboración propia } \\
\hline
\end{tabular}

De acuerdo con lo anterior para una sub-rasante con un CBR de 3,0 y una subbase granular de $0,15 \mathrm{~m}$, el módulo de reacción del conjunto sub-rasante-sub-base corresponde a $37.982 \mathrm{kN} / \mathrm{m}^{3}$.

\section{RESULTADOS DE LA EVALUACIÓN}

Con el fin de determinar los diagramas de momentos de la placa-huella para evaluar los requerimientos del acero de refuerzo se evaluó el modelo de elementos finitos con los siguientes parámetros: 
- Ancho de banda:

0,90 m (ancho de la Placa-huella)

- Resistencia a la compresión de concreto

$21.000 \mathrm{kN} / \mathrm{m}^{2}(21 \mathrm{MPa})$

- Módulo de elasticidad del concreto

$2,1 * 10^{7} \mathrm{kN} / \mathrm{m}^{2}(21.000 \mathrm{MPa})$

- Módulo de reacción del soporte

\section{$37.982 \mathrm{kN} / \mathrm{m}^{3}$}

En las figuras 7 y 8 se presentan los diagramas de deformación y momento de la placa-huella, resultantes de las cargas aplicadas por el paso de un camión C3 y el peso propio del pavimento en placa-huella.

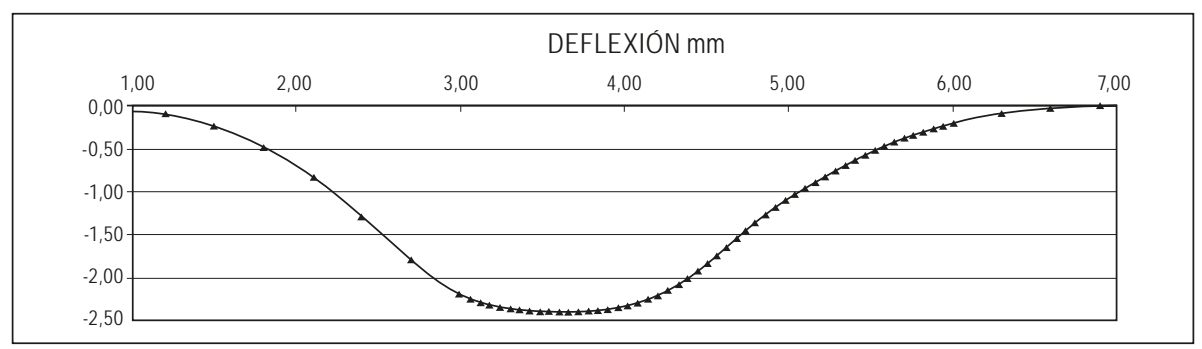

Figura 7. Deflexiones en la placa huella.

Fuente: elaboración propia

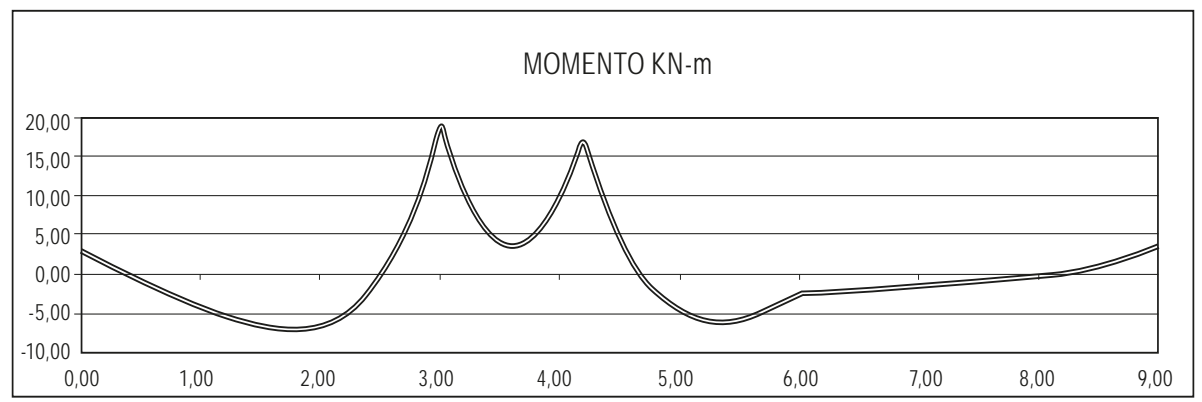

Figura 8. Momentos en la placa-huella.

Fuente: elaboración propia

La figura 8 muestra que el momento máximo que se presenta en la placa-huella corresponde a $18,4 \mathrm{kN}-\mathrm{m}$, una placa huella de $0,15 \mathrm{~m}$ de espesor, con una barra corrugada \# 4 cada $0,15 \mathrm{~m}$ tiene una capacidad por momento de $20,5 \mathrm{kN}-\mathrm{m}$. En consecuencia, este es el refuerzo recomendado debido a que la capacidad supera levemente el momento calculado. Adicionalmente, una separación de $0,15 \mathrm{~m}$ suministra una adecuada distribución de esfuerzos a lo ancho de la placa-huella.

En el caso de elementos de concreto reforzado, con elementos de concreto que están en interacción con el suelo, se recomienda un recubrimiento mínimo de 0,075 
m. Un espesor de $0,15 \mathrm{~m}$ para los pavimentos en placa-huella es el espesor mínimo aceptable que garantiza el recubrimiento mínimo, colocando el refuerzo en el eje central longitudinal. Esta ubicación del refuerzo contribuye a disminuir la fisuración, mantiene las fisuras unidas evitando que aumente su separación y ayuda a mantener la integridad de las placas evitando la separación en bloques del pavimento con placa-huella.

Se recomienda la colocación de acero transversal con barras \# 2 cada 30 centímetros, con el propósito de fijar el acero longitudinal, evitar desplazamientos durante la construcción y permitir una mejor distribución de esfuerzos transversalmente. Para el acero longitudinal de las placas-huella se recomiendan traslapos de $0,60 \mathrm{~m}$, en el tercio central y como máximo en el 50\% del acero de la sección.

En caso de requerirse placas-huella con ancho superior a los $0,90 \mathrm{~m}$, por ejemplo, en los sobreanchos de las curvas, se debe conservar el mismo acero de refuerzo, con igual distribución que en las placas-huella de $0,90 \mathrm{~m}$ de ancho. Las bermas-cuneta, por ser elementos que pueden estar sometidos a las mismas cargas que la placa-huella, deben tener un refuerzo similar al de estas.

Las riostras son elementos cuya función es confinar la sub-base y garantizar que las placas huellas trabajen monolíticamente; las mismas están sometidas a esfuerzos bajos por lo que se recomienda la colocación de la cuantía mínima de refuerzo para estos elementos que corresponde a 4 barras \# 4 longitudinalmente (dos arriba y dos abajo) y estribos \# 2 cada $15 \mathrm{~cm}$. Se recomiendan traslapos de $0,60 \mathrm{~m}$ para los aceros de las riostras.

Las cuantías de refuerzo propuestas son muy cercanas a la cuantía mínima requerida por el pavimento. La reducción de la cuantía de refuerzo implicaría un incremento en el espaciamiento de las barras \# 4, dado que con barras \# 3 la estructura no podría resistir las solicitaciones de la carga de diseño utilizada en el análisis; esto no sería conveniente porque se afectaría la distribución de esfuerzos a lo ancho de la placa-huella.

Las juntas de construcción de la placa-huella deben hacerse a un tercio de la longitud de la misma, medido desde la riostra, y en ese punto se permite traslapo de máximo el 50\% del refuerzo longitudinal. La junta de construcción de la riostra debe hacerse a $0,30 \mathrm{~m}$ del punto donde termina la placa-huella; en este punto se permite traslapo de máximo el 50\% del acero longitudinal.

\section{COMPARACIÓN CON LA SITUACIÓN ACTUAL DE LOS PAVIMENTOS EN PLACA- HUELLA EN COLOMBIA}

La comparación directa entre la estructura recomendada por la especificación 500-1P y la recomendada en el presente estudio no puede realizarse con exactitud debido a que no 
se conocen los parámetros de diseño tenidos en cuenta para el cálculo de la estructura de la especificación 500-1P; no se conoce el vehículo de diseño, ni la capacidad de soporte de la sub-rasante, parámetros determinantes en la capacidad estructural del tipo de pavimento propuesto. Al comparar la geometría y los requerimientos de acero de refuerzo de ambas estructuras, se encuentra que son menores las requeridas por la especificación 500-1P; esto hace presumir que el vehículo de diseño pudo ser inferior al vehículo $\mathrm{C} 3$, generando gran incertidumbre a la posibilidad de que por el pavimento circule este tipo de vehículo.

\section{CONCLUSIONES Y RECOMENDACIONES}

Se puede utilizar el modelo de Winkler para calcular los diagramas de momentos de pavimentos con placa huella con el fin de realizar el diseño estructural de este tipo de pavimentos. Se debe evaluar cuidadosamente la carga máxima esperada durante el período de diseño del pavimento, debido a que el diseño se realiza por carga última y una sola aplicación de una carga superior a la carga máxima de diseño podría producir fisuración excesiva en el pavimento induciendo su deterioro.

Bajo las condiciones estudiadas, el diagrama de momentos muestra que el máximo momento es de $18,4 \mathrm{kN}-\mathrm{m}$, que para una placa de $0,15 \mathrm{~m}$ de espesor requiere la cuantía mínima de refuerzo; por esta razón, la cuantía de refuerzo permanecerá constante, a pesar de que las condiciones del diseño cambien de manera favorable, como, por ejemplo, la disminución de la carga de diseño, la disminución de los factores de mayoración, el incremento del espesor de la sub-base o el incremento de la resistencia de la sub-rasante. Esto es importante teniendo en cuenta la incertidumbre que existe en la determinación de los factores de mayoración de las cargas debido a falta de referencias científicas.

Los análisis se realizaron con superficie de apoyo con un módulo de reacción del conjunto de $37.982 \mathrm{kN} / \mathrm{m}^{3}$, que corresponde a una sub-rasante con CBR de 3,0 y una sub-base granular de $0,15 \mathrm{~m}$ de espesor (tablas 1 y 2). Es decir, que para el caso de sub-rasantes con valor de CBR inferior a 3,0, se deben realizar los trabajos necesarios para garantizar que la superficie de apoyo tenga como mínimo este valor de CBR. Durante el proceso constructivo se debe garantizar el cumplimiento de estas condiciones mínimas, las cuales deben mantenerse durante el período de servicio del pavimento para garantizar la durabilidad del mismo. Tanto la sub-rasante como la sub-base deben ser debidamente compactadas. Se debe garantizar el confinamiento de la sub-base por parte de la riostra y el bordillo, por lo que estos elementos deberán proyectarse por debajo de la placa, como mínimo hasta alcanzar una profundidad igual al espesor de la sub-base $(0,15 \mathrm{~m})$. 
El efecto de una mejora en la calidad del soporte de la placa-huella representa una leve disminución en los esfuerzos, deformaciones y momentos que se presentan en los pavimentos de placa-huella reforzada. Se recomienda que esta diminución de esfuerzos se considere un factor de seguridad y no se reduzcan las cuantías del refuerzo.

Dado que la revisión de literatura realizada durante el estudio no reporta información sobre el desempeño de los pavimentos en placa-huella, se recomienda evaluar el desempeño a mediano y largo plazo de este tipo de pavimentos, lo cual permitirá realizar avances hacia mejores metodologías de diseño de este tipo de pavimentos.

\section{REFERENCIAS}

[1] Alcaldía de Sibaté en Cundinamarca, Colombia, "Placa - huella en concreto reforzado," Sibaté, 2015.

[2] Scribd.com, "Especificación 500-1P placa-huella en concreto," [En línea]. Available: https:// www.scribd.com/book/72919706/Diseno-Huellas-en-Concreto. [Último acceso: 9 Marzo 2016].

[3] A. Das, Analysis of pavement structures, New York: CRC Press, Taylor \& Francis Group, 2014.

[4] C. Van, Pavement design and evaluation: the required mathematics and applications, Brussels: Federation of the Belgian Cement Industry, 204.

[5] J. F. Arango-Londoño, "Diseño de pavimentos reforzados: evaluación de factores de carga y subresistencia por el método LRFD,” Tecno Lógicas, n 14, pp. 89-103, 2005.

[6] Ministerio de Transporte, "Límites de pesos y dimensiones de los vehículos de carga en Colombia,” Resolución 004100 Ministerio de Transporte, Bogotá DC, 2004.

[7] W. Heukelom y . A. Klomp, "Dynamic testing as a means of controlling pavements during and after construction," International conference on the structural design of asphalt pavements, vol. $203, \mathrm{n}^{\circ} 1$, pp. 667-685, 1962.

[8] Y. H. Huang, Pavement Analysis and Design, Pearson/Prentice Hall, 2004.

[9] R. G. Packard, "Thickness design for concrete highway and street pavements," Portland Cement Association, 1984. 